James Foran, Department of Mathematics, University of Missouri-Kansas

City, Kansas City, MO 64110 USA, e-mail: jforan@cctr.umkc.edu

\title{
A NOTE ON CONVERGENCE IN MEASURE
}

Convergence in measure of a sequence of functions was defined by F.Riesz in 1909. (Cf.[5] and [2] p.177 ff.) It was used to provide a proof of the completeness of $L^{p}$ (Riesz-Fischer Theorem). It is also used in the proof of the weak law of large numbers and thus is sometimes called convergence in probability.

A sequence of real valued functions $\left\{f_{n}\right\}$ defined on a measure space is said to converge in measure to a function $f$, written $f_{n} \rightarrow f$ [meas.], if for every $\epsilon>0$ one has $\lim _{n \rightarrow \infty} m\left(\left|f_{n}-f\right|>\epsilon\right)=0$. Here $m E$ is the measure of $E,(f>a)$ is $\{x: f(x)>a\}$, and all functions are presumed measurable and defined on a measure space $X$. Standard results involving limits in measure can be found in [4]. In particular, convergence in measure does not imply convergence at a single point. However, the limit is unique (up to sets of measure 0 ); the limit of the sum of two sequences which converge in measure is the sum of the limits in measure; the limit in measure of a constant times the functions in a sequence is the constant times the limit function; and the limit in measure of a sequence of measurable functions is measurable. In Munroe [4] it is proved that if $m X<\infty$ then the limit in measure of the product of two sequences is the product of the limits providing the limits in measure exist. To show that this does not hold on spaces of infinite measure, he gives the example: $f_{n}(x)=x$ and $g_{n}(x)=1 / n$ for each $x \in(0, \infty)$ where $m$ is Lebesgue measure on the line. However, it is not difficult to show that the limit in measure of the product is the product of the limits under certain conditions. In particular, if the functions $f$ and $g$ are either bounded or Lebesgue integrable and if $f_{n} \rightarrow f$ [meas.] and $g_{n} \rightarrow g$ [meas.], then $f_{n} g_{n} \rightarrow f g[$ meas $]$.

Actually, there is a more general condition which guarantees that the limit in measure of the product is the product of the limits in measure. It is given

Key Words: measure, convergence in measure

Mathematical Reviews subject classification: $26 \mathrm{~A} 42$

Received by the editors April 4, 1996 
by the following natural definition: a function $f$ is said to be almost bounded if for every $\epsilon>0$ there is a number $N$ so that $m(|f|>N)<\epsilon$.

Note that the condition of being almost bounded is equivalent to the condition that there is a number $M$ so that $m(f>M)<\infty$. It is also equivalent to the condition that $\max (-N, \min (f, N))$ approaches $f$ in measure as $N \rightarrow \infty$. And furthermore, a function $f$ is almost bounded iff $f / n \rightarrow 0$ [meas.].

In examining the convergence in measure of a product, the equation $g h=$ $(g+h)^{2} / 4-(g-h)^{2} / 4$ along with the fact that the sums, differences and constant multiples behave as expected with respect to such convergence reduces the problem to that of showing:

Proposition 1. If $f$ is almost bounded and $f_{n} \rightarrow f[$ meas. $]$, then $f_{n}^{2} \rightarrow$ $f^{2}[$ meas. $]$.

Proof. Given $1>\epsilon>0$, since $f$ is almost bounded, there is an $M>1$ so that $m(|f|>M-1)<\epsilon / 4$. Since $f_{n} \rightarrow f[$ meas. $]$ there is $N$ so that $n>N$ implies $m\left(\left|f_{n}-f\right|>\epsilon / 2 M^{2}\right)<\epsilon / 4$. Since $\left(\left|f_{n}\right|>M\right) \subset(|f|>$ $M-1) \cup\left(\left|f_{n}-f\right|>1\right)$, it follows that $m\left(\left|f_{n}\right|>M\right)<\epsilon / 2$. Then since $\left(\left|f_{n}^{2}-f^{2}\right|>\epsilon\right) \subset\left(\left|f_{n}-f\right|>\epsilon / 2 M^{2}\right) \cup\left(\left|f_{n}\right|>M\right) \cup(|f|>M)$, for $n>N$, it follows that $m\left(\left|f_{n}^{2}-f^{2}\right|>\epsilon\right)<\epsilon$ and $f_{n}^{2} \rightarrow f^{2}$ [meas.].

If $f_{n}$ approaches $f$ in measure and $f$ is almost bounded, it is not necessary that all of the $f_{n}$ be almost bounded functions, but the $f_{n}$ must be almost bounded when $n$ is sufficiently large. Also, the following converse holds:

Proposition 2. If $\left\{f_{n}\right\}$ is a sequence of almost bounded functions which approaches a function $f$ in measure, then $f$ is almost bounded.

Proof. Given $\epsilon>0$, there is $N$ so that for $n>N, m\left(\left|f_{n}-f\right|>1\right)<\epsilon$. Fix $n>N$. Then there is $M$ so that $m\left(\left|f_{n}\right|>M\right)<\epsilon$. Since $(|f|>M+1) \subset$ $\left(\left|f_{n}-f\right|>1\right) \cup\left(\left|f_{n}\right|>M\right)$, it follows that $m(|f|>M+1)<2 \epsilon$ and thus $f$ is almost bounded.

That almost bounded is the best possible condition for the function $f$ and a sequence $\left\{f_{n}\right\}$ is shown by:

Proposition 3. If $f$ is not almost bounded, there is a sequence $\left\{f_{n}\right\}$ which approaches $f$ uniformly (and hence in measure) so that $f_{n}^{2}$ does not approach $f^{2}$ in measure.

Proof. If $f$ is not almost bounded, for each natural number $N$, it follows that $m(|f|>N)=\infty$. Then there is a subsequence of the natural numbers, $\left\{N_{k}\right\}$ so that if $E_{k}=\left(N_{k}<|f| \leq N_{k+1}\right)$ then $m\left(E_{k}\right)>1$. Let $f_{n}(x)=f(x)$ if $x \notin \cup_{k=n}^{\infty} E_{k}$; for each $k \geq n$ and $x \in E_{k}$, let $f_{n}(x)=f(x)+1 / k$ if $f(x)>0$ 
and $f_{n}(x)=f(x)-1 / k$ if $f(x)<0$. Then $f_{n}$ approaches $f$ uniformly. But if $k \geq n$ and $x \in E_{k}$ then $\left|f_{n}^{2}(x)-f^{2}(x)\right|>\left|f_{n}(x)-f(x)\right| \cdot 2 N_{k}>2 N_{k} \cdot 1 / k>1$. Since $\cup_{k \geq n} E_{k}$ has infinite measure, it follows that $f_{n}^{2}$ does not approach $f^{2}$ in measure.

Clearly, the almost bounded functions defined on a metric space form a linear space of functions. There is a natural metric for the space of almost bounded functions; namely, let

$$
d(f, g)=\inf \{s: m(|f-g|>s) \leq s\} .
$$

Here, it is clear that $d(f, g)=0$ iff $f=g$ almost everywhere and that $d(f, g)=d(g, f)$ and that $f$ is almost bounded iff $d(f, 0)$ is defined (that is, is finite). That the triangle inequality holds follows from the fact that if $d_{1}=$ $d(f, h)$ and $d_{2}=d(g, h)$, then $\left(|f-g|>d_{1}+d_{2}\right) \subset\left(|f-h|>\dot{d}_{1}\right) \cup\left(|g-h|>d_{2}\right)$ and thus $m\left(|f-g|>d_{1}+d_{2}\right) \leq d_{1}+d_{2}$. Thus $d(f, g) \leq d(f, h)+d(g, h)$. This metric does not come from a norm. To see this consider Lebesgue measure on $(0, \infty)$. Let $p \geq 1$ and $f(x)=1 / x^{p}$. Then an easy calculation shows that for any real number $c, d(c f, 0)=c^{1 /(p+1)}$. Thus $d(c f, 0) \neq|c| d(f, 0)$. Finally, note that $f_{n} \rightarrow f\left[\right.$ meas.] iff $d\left(f_{n}, f\right) \rightarrow 0$. This metric is a specialization of the metric (cf.[1] p.102 ff.) which is defined on the space of all measurable functions defined on a measure space $X$ and which satisfies $f_{n} \rightarrow f[$ meas.] iff $d\left(f_{n}, f\right) \rightarrow 0$; namely, $d(f, g)=|f-g|$ where for $h=f-g$

$$
|h|=\inf _{\alpha>0} \arctan (\alpha+m(h>\alpha)
$$

An additional metric for convergence in measure on spaces of finite measure can be found in [3]. It is $d(f, g)=\int|f-g| /(1+|f-g|$.

There is really nothing special about the relationship between the operation of multiplication and convergence in measure. Indeed, if $h(x, y)$ is any continuous real valued function defined on the plane, then the following result holds:

Proposition 4. If $f_{n} \rightarrow f[$ meas. $]$ and $g_{n} \rightarrow g[$ meas. $]$ and both $f$ and $g$ are almost bounded and $h(x, y)$ is a continuous real valued function defined on the plane, then $h(f, g)$ is almost bounded and $h\left(f_{n}, g_{n}\right) \rightarrow h(f, g)[$ meas.]

Proof. Given $\epsilon>0$ there is a number $M$ so that $m(f>M)<\epsilon$ and $m(g>M)<\epsilon$. There is a number $N$ so that $h(x, y)<N$ when $|x| \leq M$ and $|y| \leq M$. Since $(h(f, g)>N) \subset(f>M) \cup(g>M), m(h(f, g)>N)<2 \epsilon$ and it follows that $h(f, g)$ is almost bounded. Now there is $\delta>0$ so that if the distance from $(x, y)$ to $\left(x^{\prime}, y^{\prime}\right)$ is less than $\delta$, then $\left|h(x, y)-h\left(x^{\prime}, y^{\prime}\right)\right|<\epsilon$. But 
$\left(\left|h\left(f_{n}, g_{n}\right)-h(f, g)\right|>\epsilon\right) \subset\left(\left|f_{n}-f\right|>\delta\right) \cup\left(\left|g_{n}-g\right|>\delta\right)$ so that the measure of this set approaches 0 as $n \rightarrow \infty$ and thus $h\left(f_{n}, g_{n}\right) \rightarrow h(f, g)[$ meas.].

Clearly, the same holds true for a continuous operation $h\left(x_{1}, x_{2}, \ldots, x_{n}\right)$ and $n$ sequences of functions converging in measure to functions $f_{1}, f_{2}, \ldots, f_{n}$.

Finally, if $h(x, y)$ satisfies a Lipschitz condition, the condition that $f$ and $g$ are almost bounded can be dropped and one can reach a conclusion analogous to that for the sum, as follows:

Proposition 5. If $f_{n} \rightarrow f[$ meas. $]$ and $g_{n} \rightarrow g$ [meas.] and $h(x, y)$ satisfies there is a number $M$ so that $\left|h(x, y)-h\left(x^{\prime}, y^{\prime}\right)\right| \leq M \cdot \operatorname{dist}\left((x, y),\left(x^{\prime}, y^{\prime}\right)\right)$, then $h\left(f_{n}, g_{n}\right) \rightarrow h(f, g)$ [meas.]

Proof. Since $\left(\left|h\left(f_{n}, g_{n}\right)-h(f, g)\right|>\epsilon\right) \subset\left(\left|f_{n}-f\right|>\epsilon / 2 M\right) \cup\left(\left|g_{n}-g\right|>\right.$ $\epsilon / 2 M)$, it follows that $m\left(\left|h\left(f_{n}, g_{n}\right)-h(f, g)\right|>\epsilon\right) \rightarrow 0$ as $n \rightarrow \infty$ so that $h\left(f_{n}, g_{n}\right) \rightarrow h(f, g)[$ meas.].

\section{References}

[1] N. Dunford and J.S chwartz, Linear Operators, Interscience Publishers, New York,1958.

[2] T. Hawkins, Lebesgue's Theory of Integration, Chelsea, The Bronx, New York, 1975.

[3] E. Hewitt and K. Stromberg, Real and Abstract Analysis, Springer Verlag, 1965.

[4] M. Munroe, Introduction to Measure and Integration, Addison-Wesley, 1953.

[5] F.Riesz, Sur les suites de fonctions mesurable, Paris Acad. Sci. C.R. 148 (1909) 1303-1305. 